\title{
Picture Credits
}

\section{Armstrong}

Fig. 1 WikiCommons, Creative Commons CC BY-SA 2.5; fig. 2 @ 2019 Oturn.com; figs. 3, 5 Public domain; fig. 4 Photograph courtesy of Avalon Fotheringham; fig. 6 Copyright Doris Leslie Blau, New York; fig. 7 Map copyright worldatlas.com; fig. 8 Image Lahore Carpet Company. Best endeavours have been made to contact the image copyright holder.

\section{Gril-Mariotte}

Figs. 1-10 Photograph by the author.

\section{Lee}

Figs. 1, 5 Photograph by N. Jansen; figs. 2, 3, 6 Public domain; fig. 4 ( ) Victoria and Albert Museum, London; fig. 7 (c) François Lauginie / Château des ducs de Bretagne - Musée d'histoire de Nantes; fig. 8 Photograph by Helen Elands.

\section{Green}

Figs. 1-8 Public domain; fig. 9 Photo: Michel Urtado / Thierry Ollivier. (@ musée du quai Branly Jacques Chirac, Dist. RMN-Grand Palais / Art Resource, NY; figs. 10, 11 Photograph by the author.

\section{Glaister}

Figs. 1a-3, 7, 8 @ Victoria and Albert Museum, London; figs. 4-6, 9 @ Trustees of the British Museum, London.

\section{Labrusse and Saou-Doufrêne}

Figs. 1-4, 11 Photograph by Bernadette Nadia Saou-Dufrêne; figs. 5, 7-10 Public domain; fig. 6 Photo: Tahar Lekael.

\section{Miller}

Fig. 1 courtesy of the author; figs. 2-4 Public domain; fig. 5, 8 Photograph by the author, 2015; fig. 6 (C) musée du quai Branly - Jacques Chirac, Dist. RMN-Grand Palais / Art Resource, NY; fig. 7 Photograph courtesy of Myriem Saaidi.

\section{Rovine}

Fig. 1: Photograph by the author; fig. 2 Source: Bibliothèque nationale de France; fig. 3 Source: gallica.bnf.fr/Bibliothèque nationale de France; figs. 4-8 Public domain; fig. 9 ๑ Trustees of the British Museum.

\section{Grillot}

Figs. 1, 2 Photograph by the author.

\section{Levin}

Figs. 1-3, 5, 6 @ 2019, Banco de Mexico Diego Rivera Frida Kahlo Museums Trust/Artists Rights Society; fig. 4 Courtesy Eric Firestone Gallery, New York, NY; fig. 7 @ Miriam Schapiro; fig. 8 (C) Yasumasa Morimura; Courtesy of the artist and Luhring Augustine, New York; fig. 9 () Sambuu Zayasaikkhan. 